\title{
5. Gizzard Actin Confers a Míagnesium Sensitivity to Skeletal Muscle Myosin at Low Ionic Strengths
}

\author{
By Takashi MIKAWA*) and Koscak MarUYAMA**) \\ (Communicated by Setsuro EBASHI, M. J. A., Jan. 12, 1981)
}

The mechanism of calcium regulation in contraction and relaxation of vertebrate smooth muscle has been a current subject of intensive studies; myosin-phosphorylation and leiotonin-control theories are in sharp conflict with each other (see reviews by Hartshorne et $a l$., 1980 and by Nonomura, 1980). It is of some interest that all the investigators supporting the myosin-phosphorylation theory use skeletal muscle actin in their studies, whereas Ebashi's group employs smooth muscle actin in the leiotonin research. It has been generally regarded that actin is functionally common regardless of its origin, although myosin is highly organ-specific.

Sekine and his collaborators first observed that actin prepared from chicken gizzard was somewhat different from chicken breast muscle actin, e.g., lower viscosity values of $\mathrm{F}$-actin solutions (Suzuki et al., 1978). Later, Drabikowski's school reported that the shorter actin filaments of some preparations from chicken gizzard was attributable to a contamination with $\beta$-actinin-like protein (StrezeleckaGolaszewska et al., 1980). Prochniewicz and Strezelecka-Golaszewska (1980) also showed some difference in the interactions of gizzard and skeletal muscle actins with skeletal muscle myosin in the presence of MgATP.

In the present communication, a simple procedure to obtain pure actin from chicken gizzard will be described and a new finding, i.e., the dependence on the $\mathrm{Mg}^{2+}$ concentration of the ATPase activity of gizzard acto-breast muscle myosin at low ionic strengths, will be reported.

Materials and methods. Chicken gizzard was minced and thoroughly washed with $50 \mathrm{mM} \mathrm{KCl}$ and $1 \mathrm{mM} \mathrm{NaHCO}$. After washing with $1 \mathrm{mM} \mathrm{NaHCO}$, the residue was homogenized by Virtis-60 at 50,000 rpm for $5 \mathrm{sec}$ and was treated three times with acetone. The acetone powder was extracted with a solution consisting of $0.2 \mathrm{mM}$ ATP, $10 \mathrm{mM}$ 2-mercaptoethanol and $2 \mathrm{mM}$ Tris- $\mathrm{HCl}, \mathrm{pH} 8.0$ at $0^{\circ} \mathrm{C}$. The extract was clarified by a centrifugation for $90 \mathrm{~min}$ at $40,000 \mathrm{rpm}$. To the supernatant, $\mathrm{KCl}$ and $\mathrm{MgCl}_{2}$ were added to give final concen-

*) Department of Pharmacology, Faculty of Medicine, University of Tokyo.

**) Department of Biology, Faculty of Science, Chiba University. 
trations of $0.6 \mathrm{M}$ and $2 \mathrm{mM}$, respectively. The solution was centrifuged to sediment F-actin, leaving tropomyosin and other contaminants in the supernatant; removal of tropomyosin from F-actin in $0.6 \mathrm{M} \mathrm{KCl}$ was first described by Spudich and Watt (1971). The resulting pellet was dialyzed against the solution used for extracting acetone powder. The dialyzed solution was clarified to remove F-actin. Preparation of myosin and actin from chicken breast muscle was carried out by the methods of Szent-Györgyi (1951) with a slight modification. Purity of actin preparations was tested by a sodium dodecyl sulfate (SDS) gel electrophoresis and by a micro-two dimensional analysis method, as will be reported by Mikawa et al.(1981). $\beta$-Actinin activity of gizzard actin preparations was checked by a flow birefringence technique (Maruyama et al., 1977).

Results. The extract of chicken gizzard acetone powder contained a number of high molecular weight proteins and tropomyosin in addition to actin (Fig. 1a). The former proteins were largely removed by a high gravity centrifugation (Fig. 1c). Tropomyosin and

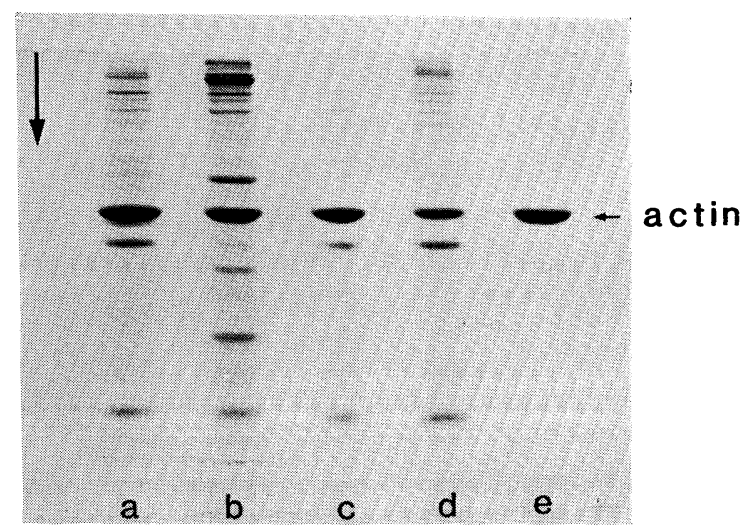

Fig. 1. SDS gel electrophoresis patterns of gizzard actin preparations. Discontinuous Tris-glycine buffer system was used. a, an original extract from acetone powder. b, precipitate of a centrifuged for $90 \mathrm{~min}$ at $40,000 \mathrm{rpm}$. c, supernatant of a. $d$, supernatant of centrifuged for $2 \mathrm{~h}$ at $40,000 \mathrm{rpm}$ in the presence of $0.6 \mathrm{M} \mathrm{KCl}$ and $2 \mathrm{mM} \mathrm{MgCl}_{2}$. e, purified actin from precipitate of $\mathrm{c}$.

a low molecular weight protein were eliminated by Spudich-Watt procedure (Fig. 1d). The final actin sample showed a single actin band in an SDS gel electrophoresis pattern (Fig. 1e). Two dimensional analysis using an isoelectric focussing and an SDS gel electrophoresis revealed that the chicken gizzard actin consisted of $\gamma$-actin together with a minor amount of $\beta$-actin in good agreement with the results of Rubenstein and Spudich (1977) and Izant and Lazarides 


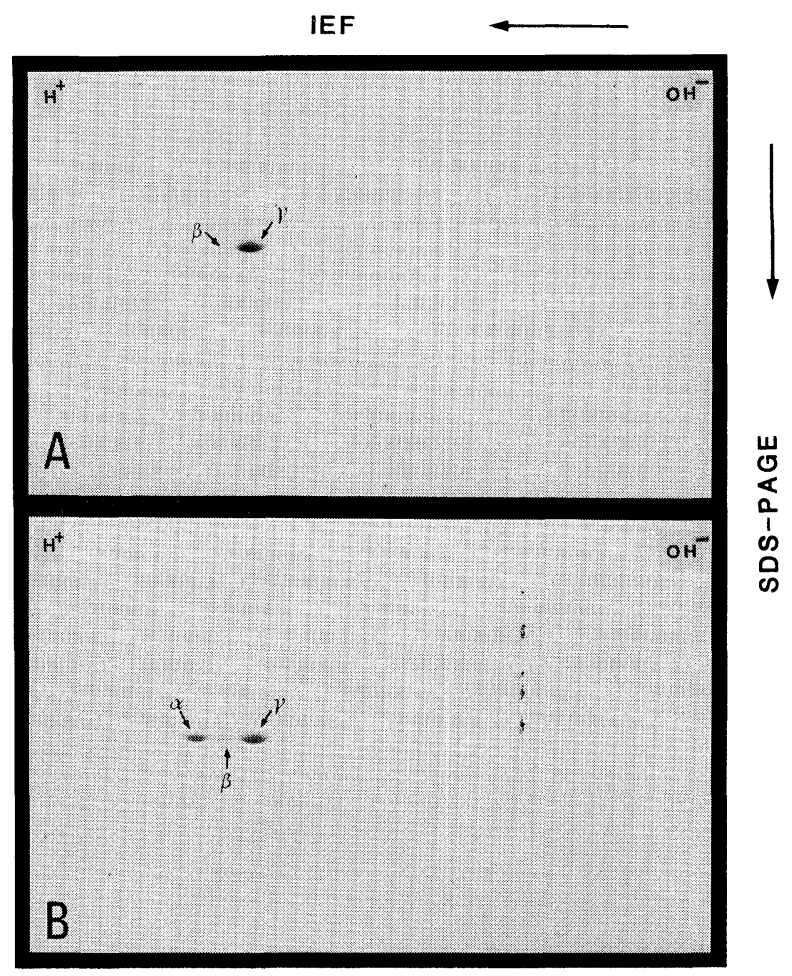

Fig. 2. Two dimensional analysis patterns of actins from chicken gizzard and breast muscle. Isoelectric focussing (IEF) was performed at $\mathrm{pH}$ ranges between 5 and 7. SDS gel electrophoresis was carried out in $13.5 \%$ polyacrylamide gels. A, gizzard actin. B, gizzard actin plus skeletal muscle actin.

(1977) (Fig. 2A). The addition of skeletal muscle actin ( $\alpha$-actin) to gizzard actin resulted in the appearance of three spots, as seen in Fig. 2B.

Flow birefringence properties of gizzard and skeletal muscle Factin solutions were compared with each other and there were not observed any differences between the two, suggesting that gizzard actin filaments were not very short in length. In fact, the intrinsic viscosity values estimated by an Ostwald type of viscometer were $7-8 \mathrm{dl} / \mathrm{g}$ for both preparations. Electron microscopic observations also showed that there were long actin filaments in both types of actin samples. To test the possible presence of $\beta$-actinin in gizzard actin preparations, the effect of sonication was investigated. Fig. 3 shows the process of association of short actin filaments fragmented under a sonic field. There was a rapid increase in the degree of birefringence due to progressive formation of long filaments by end-to-end association of the fragments in both actin samples. Had $\beta$-actinin been 


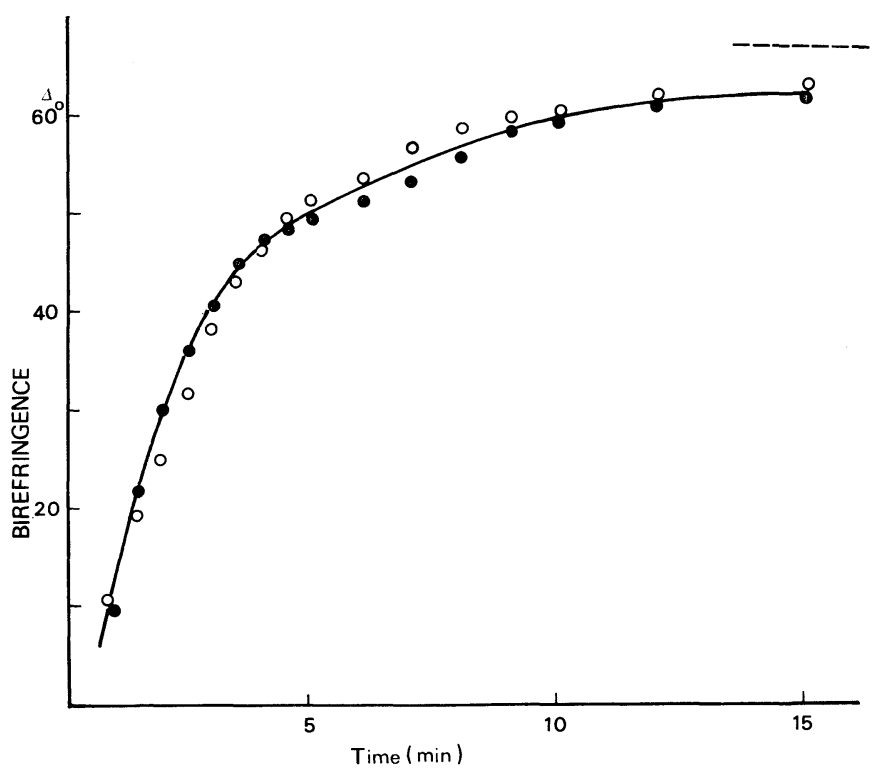

Fig. 3. Recovery process of degrees of flow birefringence of gizzard and skeletal muscle actin solutions after sonication. F-actin, $0.25 \mathrm{mg} / \mathrm{ml}$, in $0.1 \mathrm{M} \mathrm{KCl}, 0.3 \mathrm{mM} \mathrm{ATP}$ and $10 \mathrm{mM}$ Tris-HCl, $\mathrm{pH} 7.2$, was sonicated for $10 \mathrm{~s}$ in a Tomy sonifier. Birefringence $(\triangle)$ was measured in an Edsall type of apparatus at $20^{\circ} \mathrm{C}$ and velocity gradient of $100 \mathrm{~s}^{-1}$. ----, level of intact solutions. $\bullet$, gizzard actin. $\bigcirc$, skeletal muscle actin.

present, the reassociation would have been inhibited (cf. Maruyama et al., 1977). Thus, the present gizzard actin preparations were completely free of $\beta$-actinin.

The interaction of gizzard actin and skeletal muscle myosin in the presence of MgATP at low ionic strengths was observed at a molar ratio of 3 (actin/myosin) for both types of actins in the presence of $1 \mathrm{mM}$ ATP, $3 \mathrm{mM} \mathrm{MgCl}_{2}, 0.065 \mathrm{M} \mathrm{KCl}$ and $0.02 \mathrm{M}$ Tris-maleate buffer, $\mathrm{pH} 6.8$ at $25^{\circ} \mathrm{C}$. The ATPase rate was by $10 \%$ lower with gizzard actin than with skeletal actin. These results agree with those described by Prochniewicz and Strezelecka-Golaszewska (1980).

A marked difference was observed in the dependence on $\mathrm{MgCl}_{2}$ concentrations of the actomyosin ATPase activities between gizzard and skeletal muscle actins. As presented in Fig. 4, the optimal $\mathrm{MgCl}_{2}$ concentration was around $5 \times 10^{-4} \mathrm{M}$ and above this concentration the ATPase activity decreased in skeletal actomyosin. On the other hand, in gizzard acto-skeletal myosin the activity was only half of that of skeletal actomyosin at $10^{-4} \mathrm{M} \mathrm{MgCl}_{2}$, and considerably increased up to $2 \times 10^{-3} \mathrm{M}$. In other words, the optimal $\mathrm{Mg}^{2+}$ concentrations shifted to higher side in gizzard acto-skeletal myosin. This requirement of 


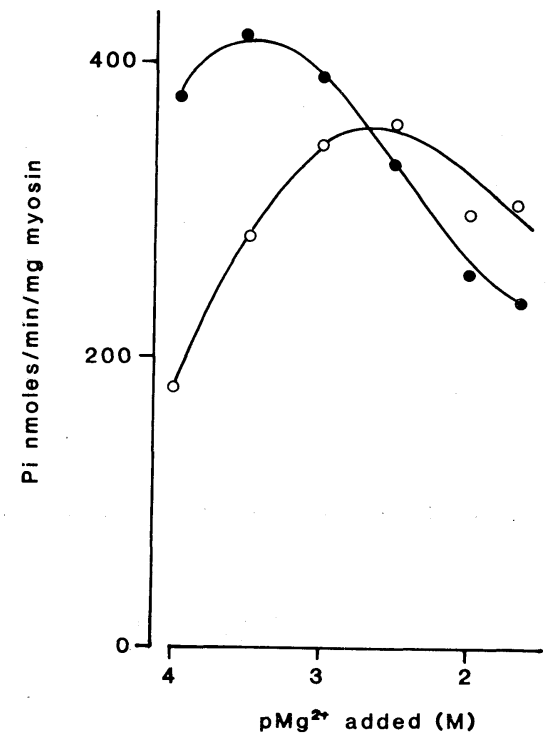

Fig. 4. Dependence on $\mathrm{MgCl}_{2}$ concentrations of the ATPase activities of the actomyosins reconstituted from chicken skeletal muscle myosin and gizzard or skeletal muscle actin. Reaction mixture contained myosin, $0.2 \mathrm{mg} / \mathrm{ml}, \mathrm{F}$-actin, $0.1 \mathrm{mg} /$ $\mathrm{ml}, 20 \mathrm{mM}$ Tris maleate buffer, $\mathrm{pH} 6.8,1 \mathrm{mM} \mathrm{ATP}, 0.056 \mathrm{M}$ $\mathrm{KCl}$ and $\mathrm{MgCl}_{2}$, as in abscissa. Incubated for $5 \mathrm{~min}$ at $25^{\circ} \mathrm{C}$. $\bigcirc$, gizzard actin. $\bullet$, skeletal muscle actin.

high concentrations of $\mathrm{Mg}^{2+}$ is a characteristic property of gizzard or smooth muscle actomyosin distinct from skeletal muscle actomyosin (Murphy et al., 1967 ; Russel, 1973).

Comments. The present actin preparations purified by a simple procedure were highly pure in criteria of SDS gel electrophoresis and isoelectric focussing (Figs. 1 and 2). Furthermore, the preparations were free of $\beta$-actinin. The interactions of gizzard actin and skeletal muscle myosin in the presence of $\mathrm{MgATP}$ at low ionic strengths were essentially the same as those of skeletal muscle actin and myosin. If we carefully examined the interactions, however, it was found that gizzard actin optimally interacted with skeletal muscle myosin at $\mathrm{MgCl}_{2}$ concentrations significantly higher than those in skeletal muscle actin and myosin (Fig. 4). Thus the requirement of high $\mathrm{M}^{2+}$ concentrations for the interaction of smooth muscle actomyosin and ATP is at least partly attributed to actin side. In this sense, it is highly desirable to use actin from vertebrate smooth muscle for the study on calcium regulation in this type of muscle.

Acknowledgements. The authors thank Professor S. Ebashi, M. J. A., for his kind interest in the present work. This study was sup- 
ported by grants from the Ministry of Education, Culture, and Science, the Ministry of Health and Welfare, and the Muscular Dystrophy Association.

\section{References}

Hartshorne, D. J., Siemankowski, R. F., and Aksoy, M. O. (1980) : Muscle Contraction. Its Regulatory Mechanisms (eds. Ebashi, S., Maruyama, K., and Endo, M.). Japan Sci. Soc. Press, Tokyo, pp. 287-302.

Izant, J. G., and Lazarides, E. (1977) : Proc. Nat. Acad. Sci. U.S.A., 74, 14501455.

Maruyama, K., Kimura, S., Ishii, T., Kuroda, M., Ohashi, K., and Muramatsu, S. (1977) : J. Biochem., 81, 215-232.

Mikawa, T., Takeda, S., and Shimizu, T. (1981): (in preparation for press).

Murphy, R. A., Bohr, D. F., and Newman, D. L. (1969): Am. J. Physiol., 217, $666-673$.

Nonomura, Y. (1980): Muscle Contraction. Its Regulatory Mechanisms (eds. Ebashi, S., Maruyama, K., and Endo, M.). Japan Sci. Soc. Press, Tokyo, pp. 329-346.

Prochniewicz, E., and Strezelecka-Golaszewska, H. (1980) : Eur. J. Biochem., 106, 305-312.

Rubenstein, P. A., and Spudich, J. A. (1977) : Proc. Nat. Acad. Sci. U.S.A., 74, $120-123$.

Russell, W. E. (1973) : Eur. J. Biochem., 33, 459-466.

Spudich, J. A., and Watt, S. (1971) : J. Biol. Chem., 246, 4866-4871.

Strezelecka-Golaszewska, H., Prochniewicz, E., Nowak, E., Zmorzynski, S., and Drabikowski, W. (1980) : Eur. J. Biochem., 104, 41-52.

Suzuki, K., Yamaguchi, M., and Sekine, T. (1978): J. Biochem., 83, 869-878.

Szent-Györgyi, A. (1951) : Chemistry of Muscle Contraction. 2nd ed., Academic Press, New York. 